\title{
Acne induzida por amineptina *
}

\author{
Acne induced by amineptine
}

\author{
Antonio Carlos Martins Guedes ${ }^{1}$ \\ Jackson Machado-Pinto ${ }^{3}$
}

\author{
Aline Almeida Bentes ${ }^{2}$ \\ Maria de Lourdes Ribeiro de Carvalho ${ }^{4}$
}

\begin{abstract}
Resumo: Relata-se um caso grave de lesões acne-símile associada a amineptina (Survector ${ }^{\circledR}$ ), proeminentes na face e dorso, acometendo outros sítios não afetados pela acne vulgar, como períneo, braços e pernas. As lesões apareceram após a auto-administração crônica de altas doses do medicamento. Lesões ceratoacantoma-símile também estavam presentes, tendo as lesões menores resposta satisfatória ao tratamento com imiquimod tópico. $O$ relato é significativo pela raridade da doença.

Palavras-chave: Acne vulgar; Ceratoacantoma; Erupção por droga

Abstract: We report one case of very severe acne-like lesions associated with amineptine (Survector ${ }^{\circledR}$ ). They were most prominent on the face and back, but were also observed on sites not affected by acne vulgaris, such as perineum, arms and legs. The lesions appeared after long-term self-medication of high doses. Keratoacanthoma-like lesions were also present, and the small ones were successfully treated with topical imiquimod. The case is significant since the disease is quite rare.

Keywords: Acne vulgaris; Drug eruptions; Keratoacanthoma
\end{abstract}

\section{INTRODUÇÃO}

A acne vulgar é inflamação das unidades pilossebáceas de algumas áreas do corpo (face, tronco e raramente na região glútea), que ocorre mais frequentemente na adolescência e apresenta formas clínicas bem definidas pela lesão preponderante: acne comedoniana, acne pápulo-pustulosa, acne nódulo-cística e acne conglobata. As lesões podem levar a cicatrizes escavadas, deprimidas e hipertróficas, sendo essas seqüelas particularmente comuns na acne nódulo-cística e na acne conglobata. A acne grave inclui manifestações nodulares inflamatórias persistentes ou recidivantes, lesões pápulo-pustulosas extensas, cicatrização exuberante e/ou fístulas. ${ }^{1}$

Em 1987 foi relatado um tipo especial de acne induzida por um antidepressivo tricíclico (amineptina). ${ }^{2}$
A acne induzida por amineptina é bastante peculiar por sua gravidade, sendo qualificada de "monstruosa" nos casos mais exuberantes; surge em idade tardia e após uso prolongado de altas doses deste antidepressivo. ${ }^{4,5}$ É forma característica e especial de acne que pode ter sua causa primária identificada ao exame clínico. Atinge preferencialmente as mulheres, na idade de involução natural da acne juvenil. É particularmente explosiva, profusa, ultrapassando os territórios habituais da acne, aparecendo essencialmente na face, pavilhão auricular, pescoço, tórax, dedos e, por vezes, nos membros e períneo. ${ }^{5-7}$

Geralmente é do tipo retencional, micro e macro-cística, tendo lesões de dois a oito milímetros, comedões fechados, brancos ou pretos e, muito raramente, inflamada. ${ }^{3,6-9}$

Recebido em 16.09.2005.

Aprovado pelo Conselho Consultivo e aceito para publicação em 26.08.07

* Trabalho realizado no Serviço de Dermatologia - Hospital das Clínicas (UFMG) - Belo Horizonte (MG), Brasil.

Conflito de interesse: Nenhum / Conflict of interest: None

Suporte financeiro: Nenhum / Financial funding: None

Professor adjunto de Dermatologia do Departamento de Clínica Médica da Faculdade de Medicina da Universidade Federal de Minas Gerais (UFMG). Doutor em Dermatologia pela UNIFESP - São Paulo (SP), Brasil.

Acadêmica de Medicina da Universidade Federal de Minas Gerais (UFMG) - Belo Horizonte (MG), Brasil

Doutor em Medicina pela Santa Casa de Belo Horizonte. Chefe da Clínica Dermatológica da Santa Casa de Belo Horizonte. Professor da Faculdade de Ciências Médicas de Minas Gerais - Belo Horizonte (MG), Brasil.

Dermatologista. Doutora em Ciências, Área de Concentração: Imunoparasitologia, do Instituto de Ciências Biológicas da Universidade Federal de Minas Gerais (ICB/UFMG) - Belo Horizonte (MG), Brasil. 
$\mathrm{Na}$ grande maioria dos casos, os doentes deprimidos consomem de dois a quatro comprimidos de amineptina por dia; trata-se de antidepressivo prescrito rotineiramente em alguns paises, como a França. ${ }^{6}$ É muito apreciado pelos pacientes por sua ação muito rápida, euforizante e tem atividade tipo anfetamina, levando a toxicomania verdadeira, por vezes dose-dependente. ${ }^{6}$

$\mathrm{O}$ tratamento da erupção acneiforme induzida pela amineptina consiste em supressão da droga, redução da toxicomania associada, abertura cirúrgica dos cistos e uso de isotretinoína $1 \mathrm{mg} / \mathrm{Kg} / \mathrm{dia}$ por tempo prolongado. ${ }^{5,6}$

\section{RELATO DE CASO}

Paciente de 52 anos, branca, sexo feminino, natural e procedente de Belo Horizonte, MG, apresentando depressão crônica, em uso de amineptina (Survector ${ }^{\circledR}$ $100 \mathrm{mg}$ ) por aproximadamente 30 anos (sic). Nos últimos anos, por automedicação, estava com uma dose de oito comprimidos ao dia. Subseqüentemente, em virtude do efeito cumulativo da droga, desenvolveu erupção acneiforme, tendo predomínio de lesões císticas, localizadas na face, região cervical, membros superiores, região glútea e vulva. Após seis anos passou a apresentar lesões ceratoacantoma-símile nas mesmas localizações (Figura 1). O quadro histopatológico das lesões ceratoacantoma-símile apresentava dilatação dos óstios das glândulas sebáceas ou écrinas preenchidas por tampões córneos e hiperplasia acentuada da camada malpighiana por possível queratinização metaplásica ou epidermóide sugerindo, por vezes, ceratoacantoma (Figura 2 e 3 ). Nessa ocasião, fez uso de isotretinoína $(1,0 \mathrm{mg} / \mathrm{Kg} / \mathrm{dia})$ e tetraciclina nas doses usuais para acne $(500$ a 1000 $\mathrm{mg} / \mathrm{dia}$ ), por aproximadamente dois anos, de forma intercalada, sem resposta terapêutica adequada. As lesões eram pruriginosas e dolorosas. As maiores foram retiradas cirurgicamente para alívio dos sintomas, e as

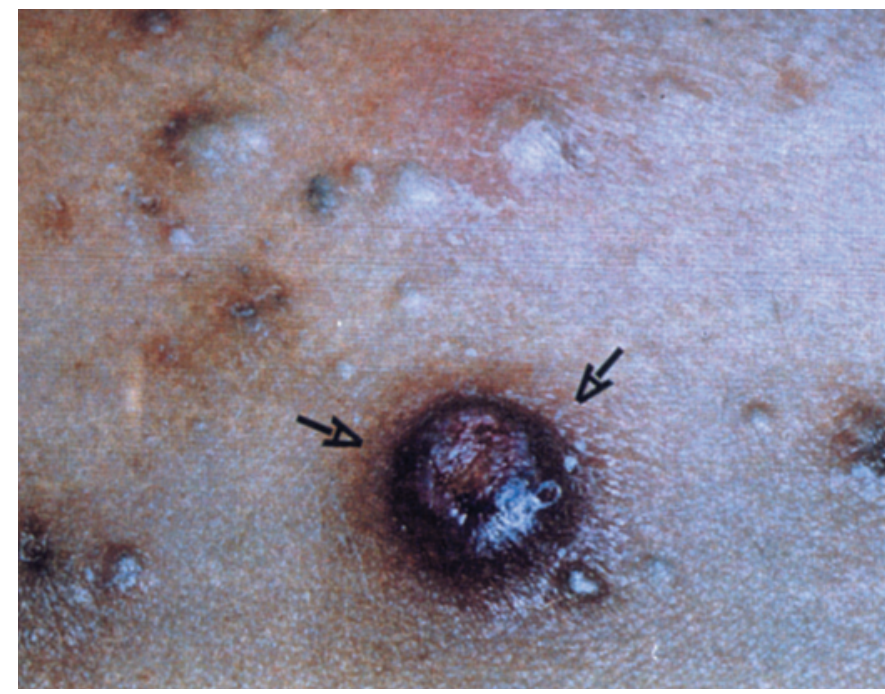

FigurA 1: Lesões micro e macrocísticas e ceratoacantoma-símile no dorso

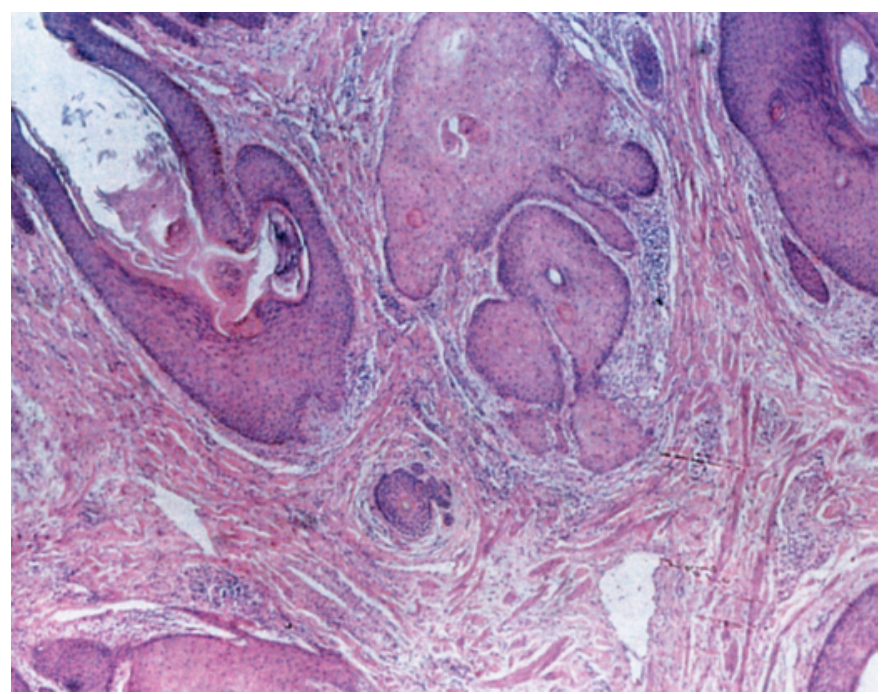

Figura 2: Aspecto histopatológico apresentando tampões córneos, lesão cística e, no centro, ilhotas de células disqueratóticas (HE 100x)

menores foram tratadas topicamente com imiquimod a $5 \%$ (3x/semana), levando à regressão clínica e deixando atrofias residuais (Figura 4 e 5). Porém, em momento algum a paciente deixou de usar amineptina. Evoluiu para o óbito devido a problemas cárdio-circulatórios. $\mathrm{O}$ exame de ressonância magnética mostrava imagens poliplóides de padrão não definido no ventrículo direito e átrios. A necropsia não foi permitida.

\section{DISCUSSÃO}

Os riscos potenciais do uso de amineptina são o desenvolvimento de lesões acneiformes, alterações neurofisiológias e dificuldade de "desmame". A acne induzida de forma iatrogênica aparece tardiamente (em média aos 42 anos de idade, extremos de 26 e 76 anos) e predomina no sexo feminino. São lesões exclusivamente do tipo retencional, micro e macrocísticas, e aparecem de

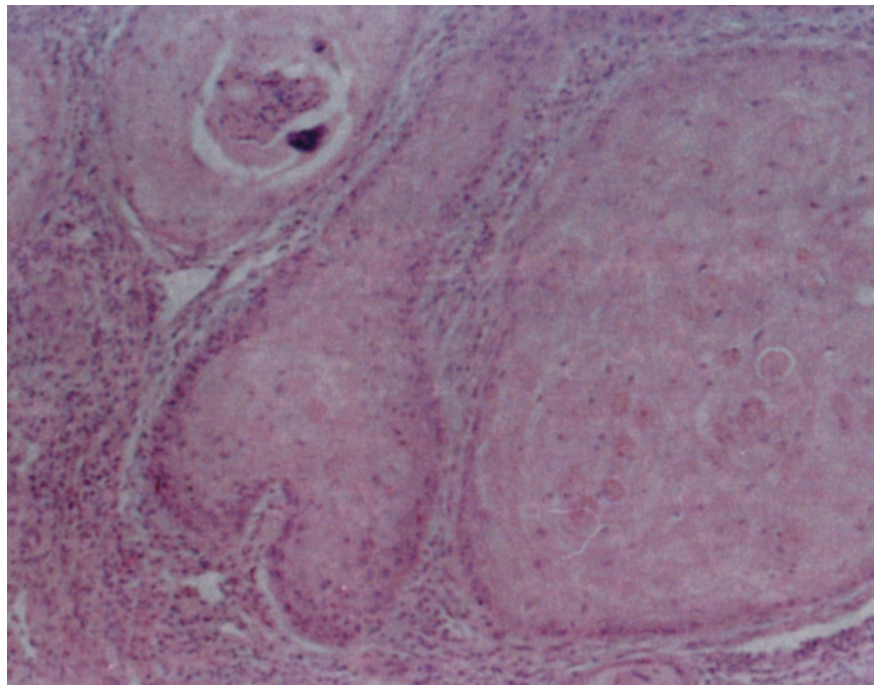

Figura 3: Detalhe de área disqueratótica com aspecto de "vidro esmerilhado", ceratoacantoma-símile (HE 400x) 

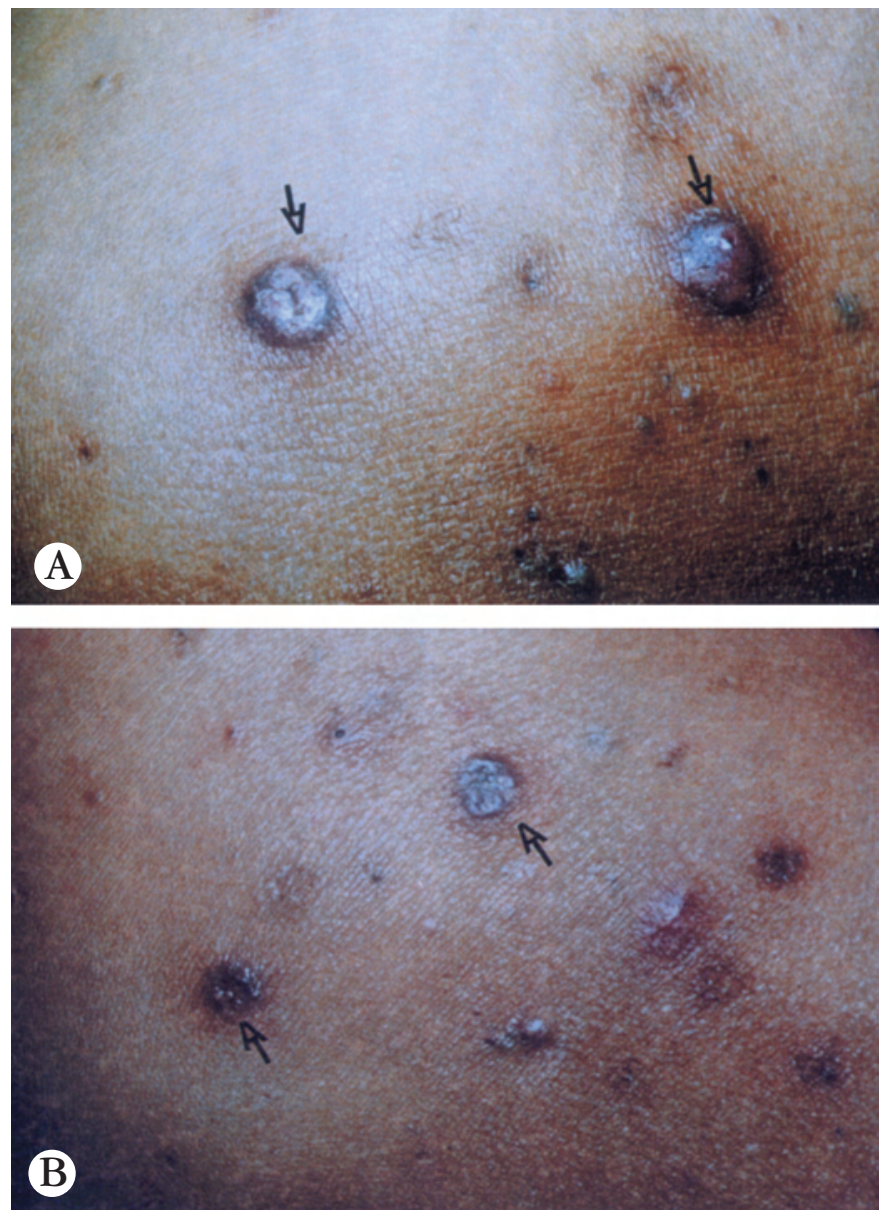

FigURA 4: Lesões ceratoacantoma-símile no dorso, antes (A) e após (B) o uso tópico de imiquimod

forma profusa e, nos casos mais graves levam a alterações faciais desfigurantes, denominadas por alguns autores de "monstruosa". Aparecem depois de meses ou anos, quando o consumo ocorre em doses supra-terapêuticas ou tóxicas. ${ }^{7,8} \mathrm{~A}$ intensidade das lesões está diretamente relacionada à dose diária e ao efeito cumulativo da medicação. ${ }^{4,5,10} \mathrm{O}$ prognóstico está relacionado com a possibilidade de um "desmame" completo e definitivo da droga. ${ }^{4}$ A análise de espectometria de massa permite identificar no plasma, na urina e até mesmo no conteúdo do cisto a presença de amineptina e seus metabólitos, o que poderia explicar a persistência da acne depois da abstinência da droga. ${ }^{4,710,11} \mathrm{O}$ medicamento se concentra não somente nas glândulas sebáceas, mas igualmente no suor. ${ }^{4}$

O ceratoacantoma é tumor de origem provável no infundíbulo folicular de crescimento rápido e predominando nas áreas expostas ao sol. É controversa a sua benignidade, pois alguns o têm como benigno com aspecto pseudomaligno, malignidade em regressão e, mesmo, uma variante do carcinoma escamoso. É afecção do idoso, com uma taxa de incidência anual de 104 / 100.000. Os ceratoacantomas podem se desenvolver em locais de trauma prévio. A maioria é do tipo "craterifor-
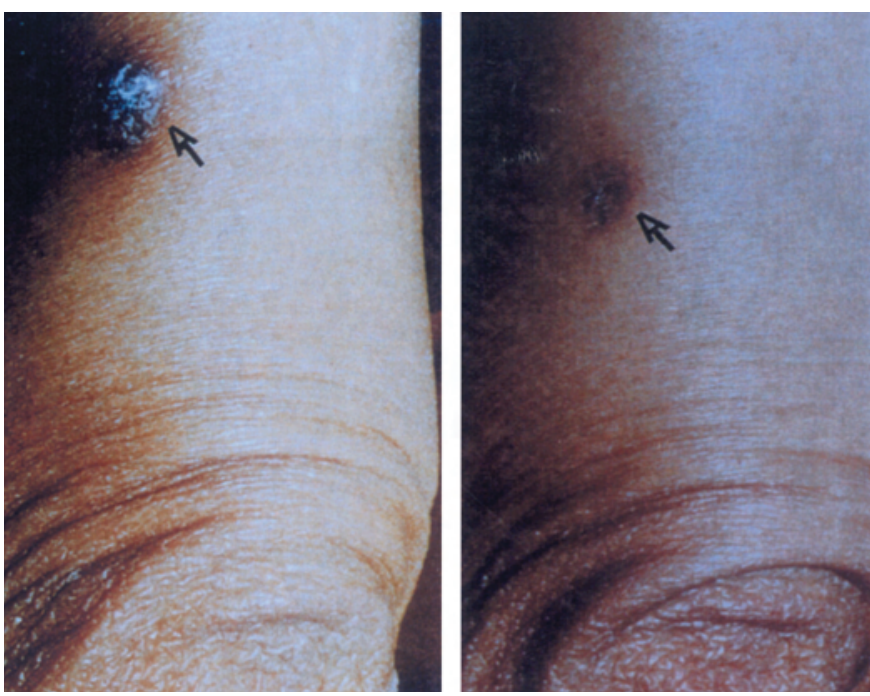

Figura 5: Lesão ceratoacantoma-símile no braço, antes (A) e após (B) o uso tópico de imiquimod

me", que cresce rapidamente, apresenta período de estado curto e então sofre regressão espontânea. Menos de $2 \%$ pertencem a variantes destrutivas, sem regressão e com crescimento invasivo persistente. ${ }^{12}$

A doente descrita, após anos de uso e de dose elevada de amineptina, mostrou múltiplas lesões semelhantes ao ceratoacantomas, tanto do ponto de vista clínico quanto histopatológico. Sendo reconhecida a origem folicular do ceratoacantoma não se poderia ser taxativo quanto à sua origem em relação a esta doente, pois as suas lesões também ocorriam em áreas sem pelos, como as palmas e plantas, bem como se associavam às dilatações tanto dos óstios das glândulas sebáceas como das écrinas preenchidas por tampões córneos. Admite-se tratar de hiperplasia acentuada da camada malpighiana por possível queratinização metaplásica ou epidermóide, lembrando ceratoacantoma. Não foi possível pesquisar metabólitos da droga no interior dessas lesões. Como a droga pode ser encontrada na secreção sebácea e no suor, haveria uma queratinização metaplásica nas lesões pápulo-queratóticas, tanto na glândula écrina (siringometaplasia) como na glândula sebácea, dando o aspecto acantótico hiperplásico e aparência vítrea. Este tipo de queratinização é benigno e não é exclusividade desta droga, pois pode ocorrer em diversas dermatoses tumorais e inflamatórias, após a administração de substâncias exógenas tóxicas como quimioterápicos (doxorubicina, cytarabine), 2,3,7,8-tetrachlorodibenzeno-p-dioxine (cloracne), pantomima (injeção de óleo), antiinflamatórios não esteróides (benaxoprofeno). $\mathrm{O}$ mecanismo de formação desta metaplasia não está bem definido, possivelmente origina-se por ação inflamatória crônica ou ação tóxica. A inflamação seria responsável por necrose celular que levaria a posterior metaplasia epitelial no canal de excreção écrina. $\mathrm{O}$ acúmulo de substâncias tóxicas, especial- 
mente medicamentos, seria responsável por efeito catalisador de diferentes tipos de processos como queratinização dos canais excretores, proliferação do epitélio ductal, hidradenite écrina neutrofílica com necrose das glândulas écrinas e queratinização associada a focos de necrose. Admite-se que haveria uma quimiotoxicidade direta sobre os anexos da pele (glândulas sebáceas e sudoríparas), colocando a amineptina com as demais drogas responsáveis por estes tipos de manifestações agrupadas como toxidermias anexiais. ${ }^{4}$

\section{REFERÊNCIAS}

1. Fitzpatrick TB, Johnson RA, Wolff $\mathrm{K}$, Suurmond D. Dermatologia: atlas e texto. 4 ed. São Paulo: McGraw-Hill; 2002. p.1041.

2. Thioly-Bensoussan D, Edelson Y, Cardinne A, Grupper CH. Acné monstrueuse iatrogène provoquée par le Survector: première observation mondiale. Nouv Dermatol. 1987;6:535-7.

3. Lèvigne V, Faisant M, Mourier C, Garcier F, Millon-Paitel M, Barthelemy $\mathrm{H}$, et al. Acné monstrueuse de Làdulte. Rôle inducteur du Survector*? Ann Dermatol Venereol. 1988,115:1184-5.

4. Huet P, Dandurand M, Joujoux JM, Amaudric F, Guillot B. L?Acné induite par L'Amineptine une toxidermie annexielle. Ann Dermatol Venereol. 1996;123:817-20.

5. Thioly-Bensoussan D, Charpentier A, Triller R, Thioly F, Blanchet $\mathrm{P}$, Tricoire $\mathrm{N}$, et al. Acné iatrogène a L'Amineptine (Survector*). Ann Dermatol Venereol. 1988;115:1177-80.

6. Grupper $\mathrm{CH}$. Une nouvelle acné iatrogène: L'Acné a L'Amineptine (Survector). Ann Dermatol Venereol. 1988;115:1174-6.

7. Vexiau P, Gourmel B, Husson C, Castot A, Rybojad M, Julien $\mathrm{R}$, et al. Lésions sévères de type acnéique induites par une intoxication chronique a L'Amineptine: a propos de 6 cas. Ann Dermatol Venereol. 1988,115:1180-2.

8. Teillac D, Weber MJ, Lowenstein W, de Prost Y. Acné au Survector. Ann Dermatol Venereol. 1988,115:1183-4.

9. Bedane C, Souyri N. Les Acnés Induites. Ann Dermatol Venereol. 1990;117:53-8.

10. Farella V, Sberna F, Knopfel B, Urso C, Difonzo EM. AcneLike eruption caused by amineptine. Int $\mathbf{J}$ Dermatol. 1996;35:892-3.
O imiquimod tópico a 5\% é droga imunomoduladora e que tem, recentemente, sido utilizada no tratamento de neoplasias cutâneas como o carcinoma basocelular e ceratoacantoma. ${ }^{13-16}$ Houve boa resposta das lesões menores ceratoacantoma-símile com o uso de imiquimod a 5\% tópico (3x/semana).

O relato desse caso é significativo por sua raridade; sendo importante para chamar a atenção para uma erupção acneiforme cujo diagnóstico etiológico pode ser facilitado pela relação com o uso abusivo de amineptina que, freqüentemente, é ocultado pelos doentes.

11. Vexiau P, Gourmel B, Julien R, Husson C, Fiet J, Puissant A, et al. Severe acne-like lesions caused by amineptine overdose. Lancet. 1988;1:585.

12. Habif TP. Dermatologia clínica: guia colorido para diagnóstico e tratamento. 4 ed. Porto Alegre: Artmed; 2005. p.1015.

13. Di Lernia V, Ricci C, Albertini G. Spontaneous regression of keratoacanthoma can be promoted by topical treatment with imiquimod cream. J Eur Acad Dermatol Venereol. 2004;18:626-9.

14. Bhatia N. Imiquimod as possible treatment for keratoacanthoma. J Drugs Dermatol. 2004;3:71-4.

15. Wee SA. Multiple eruptive keratoacanthomas, de novo. Dermatol Online J. 2004;10:19.

16. Navi D, Huntley A. Imiquimod 5 percent cream and the treatment of cutaneous malignancy. Dermatol Online J. 2004; 10:4. Review.

\author{
ENDEREÇO PARA CORRESPONDÊNCIA / MAILING ADDRESS: \\ Antonio Carlos Martins Guedes \\ Rua Padre Rolim, 515 Sala 708. Santa Efigênia \\ 30190030 - Belo Horizonte - MG \\ Tel./fax: (31) 3274-8400
}

